\title{
Announcements
}

(Requests for announcements must be received at least 3 months before publication.)

\section{Diagnostic Gynecologic Pathology}

The Department of Pathology, Massachusetts General Hospital, and Harvard Medical School will present a postgraduate course in Gynecologic Pathology under the direction of Dr. Robert H. Young with additional Harvard Medical School faculty members Dr. Debra A. Bell and Dr. Christopher P. Crum, and guest faculty, Dr. Philip B. Clement, Vancouver General Hospital, University of British Columbia. The course will be held at the San Diego Hilton Beach and Tennis Resort in San Diego, CA, January $15-17,2000$. The objective of this course is to provide a comprehensive review of gynecologic surgical pathology with special attention paid to problem areas, recent advances, and newly recognized entities. Instruction will be primarily by lecture but will also include discussion periods. Each participant will receive a comprehensive course syllabus.

The course has Category I accreditation for 22 hours CME credit by the American Medical Association. The fee for the course is $\$ 615.00$ (residents and fellows, \$470.00). For further information, contact: Department of Continuing Education, Harvard Medical School, 25 Shattuck Street, Boston, MA 02115; telephone 617-432-1525; e-mail: hmscme@hms.harvard.edu; fax 617-432-1562.

\section{Central American Society of Dermatopathology}

The Central American Society of Dermatopathology is sponsoring a program in neo-dermatology and dermatopathology in Quepos, Costa Rica, February 13-16, 2000. Enjoy sport fishing for sailfish and marlin, balmy days and beautiful beaches on the Costa Rican Pacific Coast, and discover new concepts in dermatopathology with an international faculty (A. Bernard Ackerman, M.D., New York City; Sergio Gonzales, M.D., Santiago; Helmut Kerl, M.D., Graz; Jorge Sanchez, M.D., San Juan; Wilhelm Meigel, M.D., Hamburg; Svein Helland, M.D., Oslo; Robert Jones, M.D., Birmingham; and Richard C. Miller, M.D., Tucson). Topics include: pitfalls in the diagnosis of melanomas, inflammatory diseases, mycosis fungoides, cutaneous lymphomas other than mycosis fungoides, pseudomalignancies, clinical dermatology for pathologists, abnormalities of hair, and more! Quepos, a city of 10,000 inhabitants, is located on the central pacific coast of Costa Rica. It is 20 minutes from San Jose by air. The scientific sessions will be in the afternoon and early evening, leaving the mornings free for fishing or other activities, and the late evening for a relaxed dinner. For more information, contact Eva Miller, 12931 E. Speedway Boulevard, Tucson, AZ 85748; telephone: 520-886-2858.

\section{5th World Congress on Trauma, Shock, Inflammation, and Sepsis: Pathophysiology, Immune Consequences}

This program will be held in Munich, Germany, February 29-March 4, 2000. The deadline for abstracts is October 30, 1999. For further information, contact: Dr. Eugen Faist, FACS, Department of Surgery, Ludwig-Maximillians-University Munich, Klinikum Grosshadern, Marchioninistrasse 15, $81377 \mathrm{Mu}-$ nich, Germany Tel: 49-89-7095-5461; e-mail: faist@gch.med.uni-muenchen.de; fax: 49-897095-2460.

\section{Young Investigator Research Grant}

The Society for Pediatric Pathology (SPP) is sponsoring a 1-year nonrenewable grant to be used to facilitate basic or applied research of a young investigator in the field of pediatric pathology. The following are appropriate expenditures: capital equipment, reagents and supplies, travel to meetings to present data, salary supplements, fees required to purchase specific services such as an electron microscope or other specified techniques or tests, and animal care costs.

The goals of the Young Investigator Award are to foster research within the SPP by providing seed money to young investigators who wish to pursue a career in pediatric pathology and to fund a pilot project after which the investigator can apply to other granting agencies. There will be (1) award up to a maximum of $\$ 10,000$. This is a nonrenewable, one-term award. Applicants must have an M.D. or D.O. degree and be full-time residents or fellows in an accredited pathology training program and sponsored by an SPP member or be faculty and staff who have been in pediatric pathology less than 5 years and are regular SPP members. Research into any aspect of pediatric disease will be considered, including morphologic, biochemical, chemical, and epidemiologic studies. 
To obtain an application, contact the SPP Executive Office, Degnon Associates, Inc., 6728 Old McLean Village Drive, McLean, VA 22101, 703-556-9222; fax: 703556-8729; e-mail: socpedpath@degnon.org. Please submit completed application forms to David M. Parham, M.D., Chief of Pediatric Pathology, Arkansas Children's Hospital, 800 Marshall Street, Little Rock, AR 72202-3591. Approval from either an Institutional Review Board (if human subjects are involved) and/or Animal Care Committee should be included as appropriate.

Completed applications must be received no later than March 1, 2000. They will be reviewed by the Research Committee of the SPP, and awards will be announced at the March SPP meeting. Award funding will begin on July 1, 2000. It is expected that the recipient of this award will present the results of the research at the meeting of the Society for Pediatric Pathology and submit one or more manuscripts to Pediatric and Development Pathology for consideration.

\section{Royal Brompton Hospital Imperial School of Medicine/National Heart and Lung Institute Practical Adult Cardiovascular Pathology Course}

This course, organized by Dr. M. N. Sheppard, will be held March 6-7, 2000. This particular "hands on" course approaches in detail the problems that face the diagnostic pathologist when dealing with cardiovascular pathology. The approach to a cardiac autopsy and sudden death will be emphasized. Cardiac specimens will be made available for analysis, and practical demonstrations and video demonstrations will be highlighted. A slide seminar is included. The course is aimed at trainees studying for the MRCPATH and also senior pathologists who wish to update their knowledge. Course fee: $£ 200.00$ (includes daily coffee, tea and lunch). A special fee of $£ 175.00$ for juniors in training. Contact Short Course Office, National Heart and Lung Institute, Dovehouse Street, London SW3 6LY; 44(0)2073518172; fax: 44(0)2073518246; e-mail: shortcourse.NHLI@IC.AC.UK.

\section{Combined Meeting of the Hans Popper Hepatopathology Society and the Renal Pathology Society}

The Hans Popper Hepatopathology Society and the Renal Pathology Society will have a combined scientific session in New Orleans, LA, on March 26, 2000. The course directors are Drs. Arthur H. Cohen and Stephen A. Geller. The topic will be "Disorders Affecting the Kidney and Liver Together," with seg- ments devoted to autosomal dominant polycystic kidney disease, hepatitis $\mathrm{C}$, and hepatorenal syndrome. The speakers are Dr. Wayne Grody, University of California, Los Angeles ("The Genetics of Autosomal Dominant Polycystic Kidney Disease"); Dr. Stephen A. Geller, Cedars-Sinai Medical Center, Los Angeles ("Ductal Plate Disorders of the Liver"); Dr. Glen S. Markowitz, Columbia Presbyterian Medical Center, New York ("New Animal Models of Autosomal Dominant Polycystic Kidney Disease"); Dr. Charles E. Alpers, University of Washington, Seattle ("The Kidney in Hepatitis C Infection"); Dr. Linda Ferrell, University of California, San Francisco ("Liver Biopsy Considerations Before Renal Transplantation"); and Dr. N. Kevin Krane, Tulane University ("Current Concepts of Hepatorenal Syndrome”). Information about registration can be obtained from The Secretariat, United States and Canadian Academy of Pathology, 3643 Walton Way Extension, Augusta, GA 30909; telephone: 706-7337550; e-mail: iap@uscap.usa.com; fax: 706-733-8033.

\section{Postgraduate Course in Gastrointestinal, Liver, and Pancreatic Pathology}

The Departments of Pathology of the Massachusetts General Hospital, Brigham and Women's Hospital, and Harvard Medical School will present an extensive 4-day course in gastrointestinal, liver, and pancreatic pathology under the direction of Drs. Carolyn Compton and Robert D. Odze to be held May 1-4, 2000, at the Royal Sonesta Hotel, Cambridge, Massachusetts.

The course is designed for pathologists and gastroenterologists at the resident and practitioner levels. It will provide a thorough, in-depth review of gastrointestinal, liver, and pancreatic pathology with emphasis on morphology, differential diagnosis, clinical pathologic correlation, and patient management. Emphasis will be placed on the role of the pathologist in the diagnosis and reporting of endoscopically obtained biopsy specimens and resection specimens. Instruction is primarily in the form of lectures but will also open forum discussions with a panel of expert gastrointestinal pathologists and gastroenterologists. Each registrant will receive a comprehensive course syllabus, and an optional projection slide set will be available on request.

This course meets the criteria for 28 hours of category $1 \mathrm{CME}$ credits by the American Medical Association. For further information, contact Department of Continuing Education, Harvard Medical School, 25 Shattuck Street, Boston, MA 02115; telephone: 617-432-1525; fax: 617-432-1562; e-mail: hms-cme@hms.harvard.edu. 


\section{Memorial Sloan-Kettering Cancer Center New Millennium Course in Surgical Pathology of Neoplastic Diseases}

The Department of Pathology of Memorial SloanKettering Cancer Center (MSKCC) presents the 23rd annual course on the Surgical Pathology of Neoplastic Diseases. The course will be held in the auditorium of the Rockefeller Research Laboratory building, located at 430 East 67 Street (between First and York Avenues), on the fashionable Upper East Side of Manhattan, May 1-5, 2000.

The course emphasizes both the state of knowledge of the pathology of eight disease management areas and the role of molecular pathology in tumor evaluation, at the beginning of the new century. Some of the topics discussed will include needle biopsies of the breast, role of sentinel lymph node mapping, usefulness of Her-2/neu in breast cancer, evaluation of prostatic needle biopsies, adrenal and pancreatic tumors, dysplastic nevi and malignant melanoma, recent work in bone and soft tissue pathology, and oligodendroglial tumors.

The target audience for this course is practicing anatomic pathologists and senior pathology trainees. At the course conclusion, participants should know the basics underlying the molecular biology of cancer and its current application to tumor diagnosis and evaluating disease outcome; be familiar with current concepts and new entities in the covered areas; know the main diagnostic criteria for the recognition of these entities; and have acquired up-todate knowledge of the correlation of morphologic, clinical, and molecular aspects of these entities.

The faculty will consist of members of the Department of Pathology of the Memorial Hospital, three guest pathologists in the areas of breast pathology, neuropathology, and endocrine pathology, and two Memorial clinicians. MSKCC is the oldest cancer hospital in the United States and a pioneer in the study of cancer pathology.

The registration fee is $\$ 750$ ( $\$ 500$ for pathologistsin-training; certification by Program Director required). This includes admission to all lectures, syllabus, use of a microscopic teaching slide set, breakfast buffet, refreshment breaks, and evening cocktail reception. Applications accompanied by a check for $\$ 750$ are being accepted and should be submitted as soon as possible. The fee is refundable, less a $\$ 50$ administrative charge, if cancellation is made in writing before March 31, 2000.

Despite the intense teaching schedule, an effort will be made to ensure that participants have enough time to visit restaurants and theaters. To facilitate this, the Wednesday and Friday sessions will end at 12:00 noon. If participants arrange their schedules to include a weekend, ample opportunity will exist to visit some of New York's famous cultural sites. We are not able to arrange hotel reservations, but nearby accommodations will be listed in the course brochure.

MSKCC is accredited by the Accreditation Council for Continuing Medical Education to sponsor continuing medical education for physicians. This activity meets the criteria for 28 credit hours in Category 1 of the Physician's Recognition Award of the American Medical Association, provided it is used and completed as designated.

For further information, contact Ms. Maureen Flaherty, Department of Pathology, Memorial SloanKettering Cancer Center, 1275 York Avenue, New York, NY 10021; 212-639-8338; fax: 212-772-8521.

\section{Lymph Nodes and Extranodal Sites (A Glass Slide Workshop)}

The Armed Forces Institute of Pathology and the American Registry of Pathology are cosponsoring this continuing medical education course. This highly unique glass slide workshop will be held May $4-5,2000$, at the Holiday Inn, Bethesda, Maryland. The course is specifically designed for hematopathologists, general pathologists, pathology residents, and hematology fellows. This is an excellent opportunity for the hands-on study of lesions in predominantly lymph node, spleen, and extranodal sites. The course has been expanded in volume and scope from last year. Unique study sets on hematoxylin-eosin stained slides (with pertinent immunohistochemical slides) of approximately 325 cases will be available for review, including 75 new cases. A microscope will be provided for each participant. The cases address a wide spectrum of reactive and neoplastic lesions, including the clinical history, diagnosis, and description of salient morphologic and immunophenotypic features. Short presentations will be given each hour to illustrate important diagnostic features. This provides an exceptional learning opportunity. Tuition is $\$ 378$ before April 7, 2000, $\$ 450$ afterward. Military, DoD civilians, VA, and PHS employees pay a discounted tuition of $\$ 300$. The course awards 17.5 CME credits. For more information, please contact SSgt Stephen Huntington, Department of Medical Education, Armed Forces Institute of Pathology, Washington, DC 20306-6000. Tel: 202-782-2637 or 800-577-3749 (United States only); fax: 202-7825020 or 800-441-0094 (United States only); International Toll Free Fax: 1-877-891-3482. The full course announcement will also be available on the World Wide Web at http://www.afip.org (select education, upcoming courses). 


\section{Course on Pulmonary Pathology}

This course will be held June 20-23, 2000, in London. It is designed to provide histopathologists and cytopathologists with an opportunity to study diagnostic lung pathology in a comprehensive manner. It comprises lectures and practical microscopy sessions, the latter making up roughly half the time and consisting of individual study of a unique collection of cases.

Further details and application forms are available from Professor B. Corrin, Brompton Hospital, London SW3 6NP; e-mail: b.corrin@ic.ac.uk; fax: 44-20-7351-8293.

\section{A Summerfest of Dermatopathology with Bernie}

This event will be held July 23-27, 2000, in Snowmass, CO, and will be conducted by A. Bernard Ackerman. The topic will be "Algorithmic Diagnosis of Inflammatory Skin Diseases Using Pattern Analysis Diagnosis of Adnexal Neoplasms by Silhouette: Melanoma or Not? A Fusillade of Novel Concepts About Dermatopathology." This event qualifies for CME Category 1 for 20 hours of accreditation. Contact World Travel Partners: 800-843-1110 or 520-797-1800; e-mail: Lbrown@bvtravel.com; or Paul Bozzo, M.D., 520-529-3903; e-mail: pbozzo@aol.com.

\section{1st Symposium of the International Society of Dermatopathology}

The 21st Symposium of the International Society of Dermatopathology will be held in Graz, Austria, on September 14-16, 2000. The focus of the meeting will be "Differential Diagnosis of Benign and Malignant Tumors of the Skin." In addition to formal lectures, free communications, and posters, there will be a "Self-Assessment Course in Dermatopathology of Cutaneous Tumors" conducted by an international faculty of experts in the field. One of the main topics of the meeting will be a "Tutorial on Melanocytic Lesions" consisting of 100 melanocytic tumors representing difficult cases or cases with special teaching value, to which each registrant can submit one or more cases. For more information, contact Lorenzo Cerroni, M.D., Department of Dermatology, University of Graz, Auenbruggerplatz, 8, A-8036 Graz, Austria; e-mail: lorenzo. cerroni@kfunigraz.ac.at; fax: 43-316-385-2466. 